\title{
Mathematical Modeling of Optimal Node Deployment for Indoor Wireless Sensor Networks
}

\author{
Lial Raja Missif ${ }^{1}$ and Mohammad M. Kadhum ${ }^{1,2}$ \\ ${ }^{1}$ National Advanced IPv6 Center (NAv6), Universiti Sains Malaysia (USM), 11800 Pulau Pinang, Malaysia \\ ${ }^{2}$ Telecommunication Research Laboratory, School of Computing, Queen's University, K7L 3N6 Kingston, ON, Canada \\ Email: lial@nav6.usm.my ; kadhum@nav6.usm.my ; kadhum@cs.queensu.ca
}

\begin{abstract}
Wireless Sensor Network (WSN) has been utilized in environmental monitoring where large number of connected sensor nodes collaboratively sense and report conditions of the monitoring area. Indoor event sensing and tracking is one of WSN applications that has a significant effect on saving cost economically and humanitarianly. Efficient sensor node deployment can ensure coverage, connectivity, and stability of the network. This paper presents the mathematical modeling of a node deployment for indoor wireless sensor networks where the optimal number of nodes required to satisfy the coverage of the indoor area and network connectivity requirements is determined. The proposed solution contributes to the reduction in the cost and maintenance of the network as less number of sensors is needed, compared to the traditional grid node deployment.
\end{abstract}

Index Terms-Wireless Sensor Network (WSN); Enviromental Monitoring and Tracking; Indoor Sensor Node Deployment

\section{INTRODUCTION}

Wireless sensor networks (WSN) is a promising new technology that offers huge potential for numerous applications in various domains such as environmental monitoring; medicine and healthcare; transportation; smart homes, buildings, and cities; agricultural and industrial process monitoring; military surveillance to name a few [3]. Unpredictable indoor incidents and accidents cause huge lose economically or humanitarianly[2] [1]. With their distributed control, wireless sensor technologies provide wide opportunities and promising solutions for monitoring premises to avoid and prevent the serious consequences on human and materials. Sensors can be networked to monitor an area of interest inside and outside bulidings. Buildings are equipped with WSNs for monitoring where the network can provide intelligent systems to collect data statistics from fixed sensors and process the real-time data to decide on proper on-time actions such as warning or guidance. The functionality and services provided by the indoor WSN depend on the capability and purpose of each sensor node in network. For example, sensors might be deployed at different locations in the building for monitoring heat, air-conditioning, light, and for detecting events such as fire, gas leak and explosion, or moving object, burglary, etc. Efficient tracking after the detection of an event based on robust data control and management would definitely help in making reliable distributed control decisions.

Sensor node deployment in WSNs is considered as application-dependent that can be deterministic or randomized.
In most of indoor WSNs, sensor nodes are deployed manually and are located by hand. However, in some emergency cases (e.g. after a disaster in a chemical factory) sensors are deployed randomly where they are distributed accidentally, making an ad hoc routing arrangement [11]. For many applications, the manual deployment is not practical because the access to the monitoring area might be restricted and it can take a significant amount of time as well. Deterministic deployment is feasible in situations where the sensing zone is accessible and the number of sensor nodes to be deployed is rather small [9]. In case that the subsequent scattering of nodes is inconstant, optimal clustering will be essential to cover connectivity and support operation of energy-efficient WSN. Inter sensor communication happens typically in the interior of short transmission ranges because of bandwidth and energy limitations. Thus, it is highly likely that a path will comprise multiple hops [7]. Generally, sensor nodes in a WSN take a particular view of the environment. This certain view of the sensor towards the environment is restricted in both accuracy and range. In other words, it can just cover a restricted range of the environment. Therefore, coverage is could be a crucial design factor in WSNs [12]. Sensor nodes must be connected to the base station or to each other. However, this might not stop the network topology from being adjustable and the size of the network from shrinking because of nodes failure [6]. Furthermore, connectivity is dependent on the possible random scattering of nodes [13] [8].

The aim of this paper is to propose an efficient indoor node deployment where the optimal location of sensors within the monitoring area and the number of nodes required to cover the area while ensuring connectivity are determined. The rest of the paper is organized as follows: Section 2 reviews some of the recent related work; while indoor WSN modeling is covered in Section 3. In Section 4, the proposed indoor sensor node deployment is presented. Finally, Section 5 concludes the research presented in this paper and suggests some future research works.

\section{RELATED WORKS}

This section presents some of the most recent related research works on sensor node deployment that concern monitoring area coverage and network connectivity. Bhuiyan et al. [4] addressed sensor deployment and decentralized computing and proposed a solution to deploy wireless sensors at strategic 
locations to achieve the best estimates of structural health (e.g., damage) by following the widely used wired sensor system deployment approach from civil/structural engineering. They found that faults (caused by communication errors, unstable connectivity, sensor faults, etc.) in such a deployed WSN greatly affect the performance of Structural Health Monitoring (SHM) systems. To make the WSN resilient to the faults, they presented an approach, called FTSHM (fault-tolerance in SHM), to repair the WSN and guarantee a specified degree of fault tolerance. FTSHM searches the repairing points in clusters in a distributed manner, and places a set of backup sensors at those points in such a way that still satisfies the engineering requirements. FTSHM also includes an SHM algorithm suitable for decentralized computing in the energyconstrained WSN, with the objective of guaranteeing that the WSN for SHM remains connected in the event of a fault, thus prolonging the WSN lifetime under connectivity and data delivery constraints. A novel connectivity-aware approximation algorithm for relay node placement in the WSNs is proposed by Ma et al. [10] to offer a major step forward in saving system overhead in the two-tiered WSNs. In particular, a unique local search approximation algorithm (LSAA) is introduced to solve the relay node single cover (RNSC) problem. In their algorithm, the number of added relay nodes for building the connectivity of the high-tier WSN can be significantly saved. Chen et al. [5] developed a novel maximum connected load-balancing cover tree (MCLCT) algorithm to achieve full coverage as well as BS-connectivity of each sensing node by dynamically forming load-balanced routing cover trees. Their simulation results show that the solution outperforms the existing ones in terms of energy efficiency and connectivity maintenance. In the same domain, considering the base station placement problem rather than sensor nodes (proper placement of base station has a great impact on the performance of the WSNs), Shi et al. [14] built a mathematical model for for wireless sensor networks with successive interference cancellation (SIC) to improve throughput. Although this model cannot be solved directly, it enables to identify a necessary condition for SIC on distances from sensor nodes to the base station. Based on this relationship, they proposed to divide the feasible region of the base station into small pieces and choose a point within each piece for base station placement. The point with the largest throughput is identified as the solution. Simulation results showed that the algorithm can achieve about $25 \%$ improvement compared with the case that the base station is placed at the center of the network coverage area when using SIC. B. Wang et al. [15] proposed an optimal node deployment pattern to minimize the number of nodes for completely covering a long belt. The optimal pattern uses shifted node strips for belt coverage, and they computed the best node distance, strip offset, and strip distance for different belt heights. Mathematical analysis were provided to prove its optimality in terms of the minimum node density for belt coverage. Numerical computations were used to show its superiority, compared with other well-known placement patterns such as equipartition placement. To guarantee the reliability and real time of industrial wireless sensor networks (IWSNs) from the perspective of systems, Wang et al. [16] have studies the multi-objective node placement problem. They proposed a new multi-objective node deployment model in which the reliability, real time, costs and scalability of IWSNs are addressed. Considering that the optimal node placement is an NP-hard problem, a new multi-objective binary differential evolution harmony search (MOBDEHS) was developed to tackle it, which is inspired by the mechanism of harmony search and differential evolution. The experimental results showed that the developed model is valid and can be used to design large-scale IWSNs with guaranteed reliability and real-time performance efficiently.

\section{Network Modeling}

For the purpose of efficient sensor node distribution and placement, the indoor WSN modeling can be described as follows: The area under monitoring is represented as sectors (pie slices) of a circle where each sector is divided into tracks (clusters). This circular sector modeling concept can be applied to any shape of area. Considering a rectangle area (the most common shape of indoor areas) illustrated in Fig. 1, where the circular sector radius is $R$ from angle of $\alpha$ the sector (i.e. any of the four angles of the rectangle). The circular sector model can be described by $(m+1)$ tuple $\left(m, T w_{0}, \ldots, T w_{m-1}\right)$, where $m$ is the total number of tracks in the sector, $T w_{0}$ is the width of first track, and $T w_{i}, i=1,2, \ldots, m-1$, represents the width of the $i$ th track in the sector. Let $T_{i}(i=0,1, \ldots, m-1)$ represents the $i$ th track in the sector. The width of tracks $\left(T w_{i}\right)$ is uniform (equal) for all $i=0,1, \ldots, m-1$, and it is shorter than or equal to the sensing range $\left(s r_{i}\right)$ of a sensor node $i$; that is, $T w_{i} \leq s r_{i}$. As the application of this network is kind of data logging applications for monitoring environmental changes (temperature and fire), the powerful base station (sink) is located out of the monitoring area where it is approachable by the transmission range of the sensor $\left(t r_{i}\right)$. The indoor area of interest is monitored continuously by homogeneous sensor nodes that sense the environment and sends data of $L$ bits of per unit of time directly to the base station; hence, no data aggregation or routing is assumed to be done at any sensor node in the network. Having a fixed sector radius of $R$ and a fixed number of uniform tracks in the sector $m$, then, the following property is given: $T w_{i}<s r_{i}<t r_{i}$. 


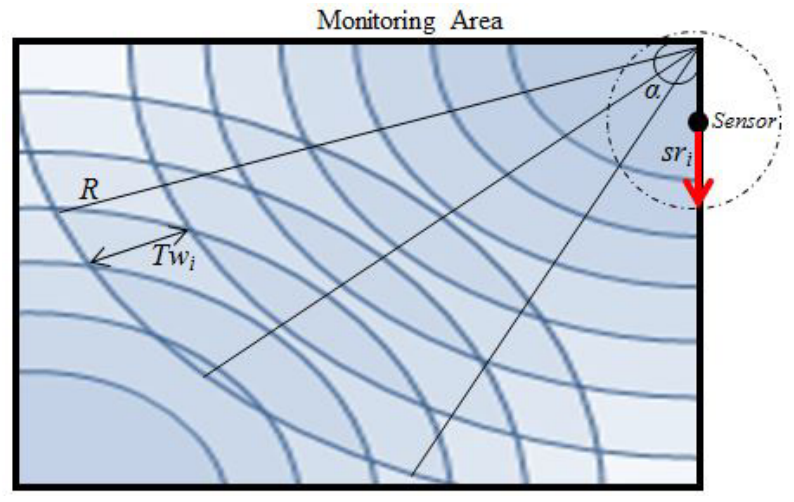

Figure 1. Monitoring area modeled by sectors for sensors deployment

\section{Proposed Indoor SEnsor Node Deployment}

In this section, the proposed sensor node distribution strategy is presented where the optimal number of sensor nodes required to satisfy the area coverage and network connectivity requirements is determined.

Proposition 1. An optimal sensor location within track $T_{i}$ of a sector can be determined such that a maximum sensing coverage of the track is achieved. Let the optimal location of a sensor be at distance $h_{i}$ away from the angle $\alpha$ of the sector, then for a circular sector model $\left(m, T w_{0}, \ldots, T w_{m-1}\right)$ with $s r_{i}, i=0,1, \ldots, m-1$, representing the sensing range of a sensor located in $T_{i}$, and that $\frac{1}{2} T w_{i} \leq s r_{i} \leq$ $\sqrt{2}\left(\sum_{j=0}^{i} T w_{j}\right), d_{i}$ for all $i=0,1, \ldots, m-1$, should meet the following condition:

$$
h_{i}= \begin{cases}\sqrt{\frac{\left(T w_{0}\right)^{2}-\left(s r_{0}\right)^{2}}{3}} & , \frac{T w_{0}}{2} \leq s r_{0} \leq T w_{0} \\ \sqrt{\left(T w_{0}\right)^{2}-\left(s r_{0}\right)^{2}} & , T w_{0}<s r_{0} \leq \sqrt{2} T w_{0}\end{cases}
$$

Proof: Assume that a sensor $i\left(s_{i}\right)$ is placed at point $\left(0, h_{i}\right)$, where $h_{i} \geq 0$. Considering the innermost track $\left(T_{0}\right)$ illustrated in Figure 2, Proposition (1) can be proven by finding the location of the sensor so that the coverage area within the track (area Ain the figure) is maximized.

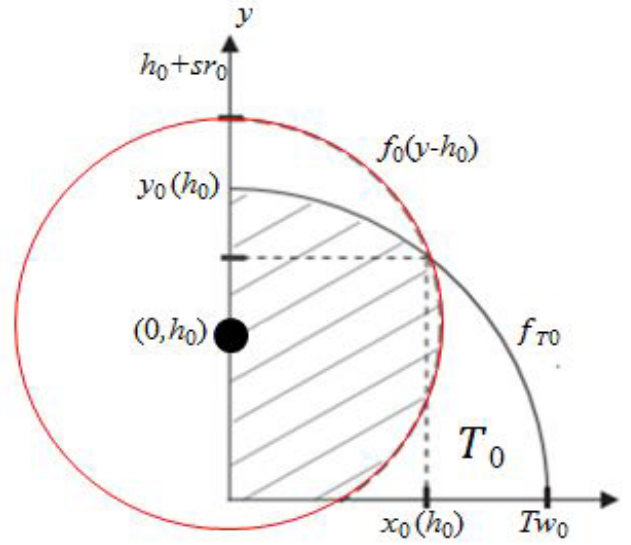

Figure 2. Optimal location of sensor in the first track $T_{0}$ of the first sector in the first quadrant of unit circle

Proof: Virtulizing two cirecles, one that includes $T_{0}$, represented by Equation (2), and another one includes the semicircle of the sensor coverage area represented by Equation (3) as follows:

$$
\begin{gathered}
x^{2}+y^{2}=T w_{0}^{2} \\
x^{2}+\left(y-h_{0}\right)^{2}=s r_{0}^{2}
\end{gathered}
$$

Solving for $x$ in Equation (1) yields:

$$
x^{2}=T w_{0}^{2}-y^{2}
$$

Subsituting (4) in (3) gives the $y_{0}\left(h_{0}\right)$ axis of the intersection point $\left(x_{0}\left(h_{0}\right), y_{0}\left(h_{0}\right)\right)$ of the two circles in the first quadrant $(\pi / 3)$ of the of the unit circle, which is:

$$
y_{0}\left(h_{0}\right)=\left(\left(T w_{0}\right)^{2}+\left(h_{0}\right)^{2}-\left(s r_{0}\right)^{2}\right) / 2 h_{0}
$$

This axis can also be achived by subtracting the two circles equations and expanding to obtain a linear equation for $x_{0}\left(d_{0}\right)$ and $y_{0}\left(h_{0}\right)$, which is the equation of the line that passes through the intersection points, as the two circles intersect. Let $f\left(h_{0}\right)$ represents area A shown in Figure 2, such that:

$$
\begin{gathered}
f\left(h_{0}\right)=\int_{0}^{y_{0}\left(h_{0}\right)} f_{0}\left(y-h_{0}\right) d y+\int_{y_{0}\left(h_{0}\right)}^{T w_{0}} f_{T_{0}}(y) d y \\
f\left(h_{0}\right)=\int_{-h_{0}}^{y_{0}\left(h_{0}\right)-h_{0}} f_{0}(y) d y+\int_{y_{0}\left(h_{0}\right)}^{T w_{0}} f_{T_{0}}(y) d y
\end{gathered}
$$

As both of $f_{0}(y)$ and $f_{T_{0}}(y)$ are contineous functions, they have antiderivatives of $F_{0}(y)$ and $F_{T_{0}}(y)$ respectively; thus, $f\left(h_{0}\right)$ can be as follows:

$$
\begin{aligned}
f\left(h_{0}\right)= & F_{0}\left(y_{0}\left(h_{0}\right)-h_{0}\right)-F_{0}\left(-h_{0}\right)+F_{T_{0}}\left(T w_{0}\right) \\
& -F_{T_{0}}\left(y_{0}\left(h_{0}\right)\right)
\end{aligned}
$$


The first derivative of $f\left(h_{0}\right)$, which is essentially the slope of the tangent line at the function, can be given as follows:

$$
\begin{aligned}
f^{\prime}\left(h_{0}\right)= & f_{0}\left(y_{0}\left(h_{0}\right)-h_{0}\right)\left(\frac{d\left(y_{0}\left(h_{0}\right)\right.}{d h_{0}}-1\right) \\
& +f_{0}\left(-h_{0}\right)-f_{T_{0}}\left(y_{0}\left(h_{0}\right)\right)\left(\frac{d\left(y_{0}\left(h_{0}\right)\right.}{d h_{0}}\right) \\
f^{\prime}\left(h_{0}\right)= & f_{0}\left(-h_{0}\right)-f_{0}\left(y_{0}\left(h_{0}\right)-h_{0}\right)
\end{aligned}
$$

which holds as $x_{0}\left(h_{0}\right)=f_{T_{0}}\left(y_{0}\left(h_{0}\right)\right)=f_{0}\left(y_{0}\left(h_{0}\right)-\right.$ $\left.h_{0}\right)$. Clearly, $f^{\prime}\left(h_{0}\right)=0$, implying that $f^{\prime}\left(h_{0}\right)=$ $\sqrt{\left(\left(T w_{0}\right)^{2}-\left(s r_{0}\right)^{2}\right) / 3}$ when $\frac{T w_{0}}{2} \leq s r_{0} \leq T w_{0}$, or $\sqrt{\left(T w_{0}\right)^{2}-\left(s r_{0}\right)^{2}}$ when $T w_{0}<s r_{0} \leq \sqrt{2} T w_{0}$. Thus, Proposition (1) is proven. It can be proven further by taking the the second derivative of $f\left(h_{0}\right)$, such that $f^{\prime \prime}\left(h_{0}\right)<0$.

Proposition 2. For a circular sector model $\left(m, T w_{0}, \ldots, T w_{m-1}\right)$, the coverage area of a sensor sensing range $s r_{i}\left(\right.$ such that $\frac{1}{2} T w_{i} \leq s r_{i} \leq \sqrt{2}\left(\sum_{j=0}^{i} T w_{j}\right)$, for $i=$ $0,1, \ldots, m-1)$, that is out of the track width $T w_{i}$ can be minimized if the optimal location of the sensor at distance $h_{i}$ away from the angle $\alpha$ of the sector satisfy the following condition:

$$
h_{i}=\sqrt{\left(\left(\sum_{j=0}^{i-1} T w_{j}\right)^{2}+\left(\sum_{j=0}^{i} T w_{j}\right)^{2}-2\left(s r_{i}\right)^{2}\right) / 2}
$$

Proof: In Figure 3, the shaded area represented by $f\left(h_{i}\right)$, where $i \geq 1$, can be expressed as follows:

$$
\begin{aligned}
f\left(h_{i}\right)= & \int_{y_{1}\left(h_{i}\right)}^{h_{i}+s r_{i}} f_{i}\left(y-h_{i}\right) d y-\int_{y_{1}\left(h_{i}\right)}^{\sum_{j=0}^{i} T w_{j}} f_{T_{i}}(y) d y \\
& +\int_{h_{i}-s r_{i}}^{y_{0}\left(h_{i}\right)} f_{i}\left(y-h_{i}\right) d y+\int_{y_{0}\left(h_{i}\right)}^{\sum_{j=0}^{i-1} T w_{j}} f_{T_{i-i}}(y) d y \\
f\left(h_{i}\right)= & \int_{y_{1}\left(h_{i}\right)-h_{i}}^{s r_{i}} f_{i}(y) d y-\int_{y_{1}\left(h_{i}\right)}^{\sum_{j=0}^{i} T w_{j}} f_{T_{i}}(y) d y \\
& +\int_{-s r_{i}}^{y_{0}\left(h_{i}\right)} f_{i}(y) d y+\int_{y_{0}\left(h_{i}\right)}^{\sum_{j=0}^{i-1} T w_{j}} f_{T_{i-i}}(y) d y
\end{aligned}
$$

where

$$
\begin{gathered}
f_{i}(y)=\sqrt{\left(s r_{i}\right)^{2}-(y)^{2}} \\
f_{T_{i-1}}(y)=\sqrt{\left(\sum_{j=0}^{i-1} T w_{j}\right)^{2}-(y)^{2}} \\
y_{0}\left(h_{i}\right)=\frac{\left(\sum_{j=0}^{i-1} T w_{j}\right)^{2}+\left(h_{i}\right)^{2}-\left(s r_{i}\right)^{2}}{2 h_{i}}
\end{gathered}
$$

$$
y_{1}\left(h_{i}\right)=\frac{\left(\sum_{j=0}^{i} T w_{j}\right)^{2}+\left(h_{i}\right)^{2}-\left(s r_{i}\right)^{2}}{2 h_{i}}
$$

Let $F_{i}(y), F_{T_{i}}(y)$, and $F_{T_{i-1}}(y)$ represent the antiderivatives of $f_{i}(y), f_{T_{i}}(y)$, and $f_{T_{i-1}}(y)$ respectively; then $f\left(h_{i}\right)$ can be as follows:

$$
\begin{aligned}
f\left(h_{i}\right)= & F_{i}\left(s r_{i}\right)-F_{i}\left(y_{1}\left(h_{i}\right)-h_{i}\right)-F_{T_{i}}\left(\sum_{j=0}^{i} T w_{j}\right) \\
& +F_{i}\left(y_{0}\left(h_{i}\right)-h_{i}\right)-F_{i}\left(-s r_{i}\right) \\
& +F_{T_{i-1}}\left(\sum_{j=0}^{i-1} T w_{j}\right) \\
& +F_{T_{i}}\left(y_{1}\left(h_{i}\right)\right)-F_{T_{i-1}}\left(\left(y_{1}\left(h_{i}\right)\right)\right.
\end{aligned}
$$

The first derivative of $f\left(h_{i}\right)$ can be given as follows:

$$
\begin{aligned}
& f^{\prime}\left(h_{i}\right)= \frac{d F_{i}\left(s r_{i}\right)}{d\left(s r_{i}\right)} \frac{d\left(s r_{i}\right)}{d\left(h_{i}\right)} \\
&-\frac{d F_{i}\left(y_{1}\left(h_{i}\right)-h_{i}\right)}{d\left(y_{1}\left(h_{i}\right)-h_{i}\right)} \frac{d\left(y_{1}\left(h_{i}\right)-h_{i}\right)}{d\left(h_{i}\right)} \\
&-\frac{d F_{T_{i}}\left(\sum_{j=0}^{i} T w_{j}\right)}{d\left(\sum_{j=0}^{i} T w_{j}\right)} \frac{d\left(\sum_{j=0}^{i} T w_{j}\right)}{d\left(h_{i}\right)} \\
&-\frac{d F_{i}\left(-s r_{i}\right)}{d\left(-s r_{i}\right)} \frac{d\left(-s r_{i}\right)}{d\left(h_{i}\right)} \\
&+\frac{d F_{T_{i-1}}\left(\sum_{j=0}^{i-1} T w_{j}\right)}{d\left(\sum_{j=0}^{i-1} T w_{j}\right)} \frac{d\left(\sum_{j=0}^{i-1} T w_{j}\right)}{d\left(h_{i}\right)} \\
&+\frac{d F_{T_{i}}\left(y_{1}\left(h_{i}\right)-h_{i}\right)}{d\left(y_{1}\left(h_{i}\right)\right.} \frac{d y_{1}\left(h_{i}\right)}{d\left(h_{i}\right)} \\
&-\frac{d F_{T_{i-1}}\left(y_{0}\left(h_{i}\right)-h_{i}\right)}{d\left(y_{0}\left(h_{i}\right)\right.} \frac{d y_{0}\left(h_{i}\right)}{d\left(h_{i}\right)} \\
& f^{\prime}\left(h_{i}\right)=\left(f _ { T _ { i } } \left(y_{1}\left(h_{i}\right)-f_{i}\left(y_{1}\left(h_{i}\right)-h_{i}\right) \frac{d y_{1}\left(h_{i}\right)}{d\left(h_{i}\right)}\right.\right. \\
&\left.+f_{i}\left(y_{0}\left(h_{i}\right)-h_{i}\right)-f_{T_{i-1}}\left(y_{0}\left(h_{i}\right)\right)\right) \frac{d y_{0}\left(h_{i}\right)}{d\left(h_{i}\right)} \\
&+f_{i}\left(y_{1}\left(h_{i}\right)-h_{i}\right)-f_{i}\left(y_{0}\left(h_{i}\right)-h_{i}\right) \\
& f_{i}\left(y_{1}\left(h_{i}\right)-h_{i}\right)-f_{i}\left(y_{0}\left(h_{i}\right)-h_{i}\right)
\end{aligned}
$$

Equation (21) holds as

$$
f_{i}\left(y_{1}\left(h_{i}\right)-h_{i}\right)=f_{T_{i}}\left(y_{1}\left(h_{i}\right)\right)=x_{2}\left(h_{i}\right)
$$

and

$$
f_{i}\left(y_{0}\left(h_{i}\right)-h_{i}\right)=f_{T_{i-1}}\left(y_{0}\left(h_{i}\right)\right)=x_{1}\left(h_{i}\right)
$$

Thus, $f^{\circ}\left(h_{i}\right)=0$ which results in that $h_{i}=\sqrt{\left(\left(\sum_{j=0}^{i-1} T w_{j}\right)^{2}+\left(\sum_{j=0}^{i} T w_{j}\right)^{2}-2\left(s r_{i}\right)^{2}\right) / 2}$, which proves Proposition 2. 


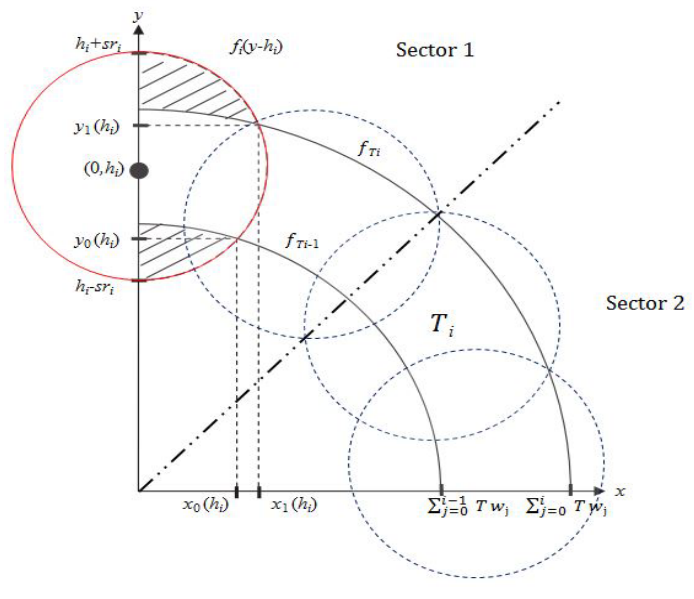

Figure 3. Optimal location of sensor in $T_{i}$, where $i \geq 2$

Proposition 3. For a circular sector model $\left(m, T w_{0}, \ldots, T w_{m-1}\right)$ with a fixed width $T w_{i}$ of unifom tracks, and a fixed distance $h_{i}$ of sensor location from the angle $\alpha$ of the sector, knowning the sensor sensing range $s r_{i}$, the number of sensors that are needed to cover the ith track $\left(N T_{i}\right)$, for all $i=0,1, \ldots, m-1$, of the sector is:

$$
\begin{aligned}
N T_{i}= & \left\lceil\frac{\pi / 4}{2 \cos ^{-1}\left(\frac{\left(h_{i}\right)^{2}+\left(\sum_{j=0}^{i} T w_{j}\right)^{2}-\left(s r_{i}\right)^{2}}{2 h_{i}\left(\sum_{j=0}^{i} T w_{j}\right)}\right.}\right\rceil \\
& \text { for } i=0,1, \ldots, m-1
\end{aligned}
$$

Proof: Consider the two neighboring nodes located in $T w_{i}$ as shown in Figure 4.

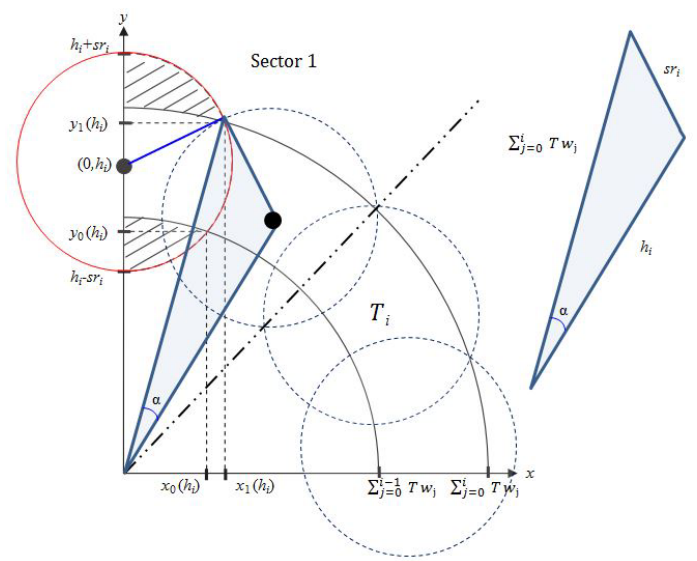

Figure 4. Maximum angle $\alpha$ between two neighboring nodes to ensure the coverage and connectivity in the track

A maximum coverage area in the $i$ th track can be achieved by where no gap between those nodes (with respect to the track width $T w_{i}$ ) is exist. Cosine law helps in derving the maximum angle $\alpha$ of the two neighboring nodes placed in $T w_{i}$, as follows:

$$
\left(s r_{i}\right)^{2}=\left(h_{i}\right)^{2}+\left(\sum_{j=0}^{i} T w_{j}\right)^{2}-2 h_{i}\left(\sum_{j=0}^{i} T w_{j}\right) \cos (\alpha)
$$

Hence,

$$
\alpha_{i}=\frac{\left(h_{i}\right)^{2}+\left(\sum_{j=0}^{i} T w_{j}\right)^{2}-\left(s r_{i}\right)^{2}}{2 h_{i}\left(\sum_{j=0}^{i} T w_{j}\right)}
$$

Considering that the first quadrant of unit circle is of two sectors, thus, the angel of each secotr is $45^{\circ}$. Therefore, the number of sensors that are needed to cover the $i$ th track is $N T_{i}=\left\lceil(\pi / 4) /\left(2 \cos ^{-1}\left(\frac{\left(h_{i}\right)^{2}+\left(\sum_{j=0}^{i} T w_{j}\right)^{2}-\left(s r_{i}\right)^{2}}{2 h_{i}\left(\sum_{j=0}^{i} T w_{j}\right)}\right)\right)\right]$ for all $i=0,1, \ldots, m-1$.

\section{CONCLUSION AND FUTURE WORK}

In this paper, an efficient node distribution solution for indoor Wireless sensor networks (WSN) that maintains the coverage of the monitoring area and network connectivity has been proposed. The indoor WSN has been modeled using circular sector modeling concept, where the tracks of the sector are of a uniform width. In order to verify the proposed solution, we have mathematically demonstrated how the optimal location of a sensor within a track of the sector can be achieved so that a maximum sensing coverage of the track is obtained with the minimum number of sensors. For future work, we are going to utilize the proposed solution in simulating an indoor WSN for sensing and tracking of an event such as explosion, fire, or gas.

\section{ACKNOWLEDGMENTS}

This work was supported by National Advanced IPv6 Centre of Excellence (NAv6), Universiti Sains Malaysia (USM).

\section{REFERENCES}

[1] F. Aghaeipoor, M. Mohammadi, and V.S. Naeini. Target tracking in noisy wireless sensor network using artificial neural network. In Telecommunications (IST), 2014 7th International Symposium on, pages 720-724, Sept 2014.

[2] M. Akter, M.O. Rahman, M.N. Islam, and M.A. Habib. Incremental clustering-based object tracking in wireless sensor networks. In Networking Systems and Security (NSysS), 2015 International Conference on, pages 1-6, Jan 2015.

[3] Giuseppe Anastasi, Marco Conti, Mario Di Francesco, and Andrea Passarella. Energy conservation in wireless sensor networks: A survey. Ad hoc networks, 7(3):537-568, 2009.

[4] M. Z. A. Bhuiyan, G. Wang, J. Cao, and J. Wu. Deploying wireless sensor networks with fault-tolerance for structural health monitoring. IEEE Transactions on Computers, 64(2):382-395, Feb 2015.

[5] C. P. Chen, S. C. Mukhopadhyay, C. L. Chuang, M. Y. Liu, and J. A. Jiang. Efficient coverage and connectivity preservation with load balance for wireless sensor networks. IEEE Sensors Journal, 15(1):48-62, Jan 2015.

[6] Wenliang Du, Jing Deng, Yunghsiang S Han, Shigang Chen, and Prainod K Varshney. A key management scheme for wireless sensor networks using deployment knowledge. In INFOCOM 2004. Twentythird AnnualJoint conference of the IEEE computer and communications societies, volume 1. IEEE, 2004. 
[7] A.K.M.M. Islam, S. Baharun, and K. Wada. An overview on dynamic wireless sensor network architectures. In Informatics, Electronics Vision (ICIEV), 2012 International Conference on, pages 464-468, May 2012.

[8] S. Lall, A. S. Alfa, and B. T. Maharaj. Optimal placement and power allocation for jammers in wireless mesh networks. In Vehicular Technology Conference (VTC Fall), 2015 IEEE 82nd, pages 1-7, Sept 2015.

[9] Xiaoxi Liu, Ruiying Li, and Ning Huang. A sensor deployment optimization model of the wireless sensor networks under retransmission. In Cyber Technology in Automation, Control, and Intelligent Systems (CYBER), 2014 IEEE 4th Annual International Conference on, pages 413-418. IEEE, 2014.

[10] C. Ma, W. Liang, M. Zheng, and H. Sharif. A connectivity-aware approximation algorithm for relay node placement in wireless sensor networks. IEEE Sensors Journal, 16(2):515-528, Jan 2016.

[11] Markus Quaritsch, Karin Kruggl, Daniel Wischounig-Strucl, Subhabrata Bhattacharya, Mubarak Shah, and Bernhard Rinner. Networked uavs as aerial sensor network for disaster management applications. $e \& i$ Elektrotechnik und Informationstechnik, 127(3):56-63, 2010.

[12] N. Roseveare and B. Natarajan. Distributed tracking with energy management in wireless sensor networks. Aerospace and Electronic Systems, IEEE Transactions on, 48(4):3494-3511, October 2012.

[13] Sanjay Shakkottai, Rayadurgam Srikant, and Ness B Shroff. Unreliable sensor grids: Coverage, connectivity and diameter. Ad Hoc Networks, 3(6):702-716, 2005

[14] Lei Shi, Jianjun Zhang, Yi Shi, Xu Ding, and Zhenchun Wei. Optimal base station placement for wireless sensor networks with successive interference cancellation. Sensors, 15(1):1676-1690, 2015.

[15] B. Wang, H. Xu, W. Liu, and L. T. Yang. The optimal node placement for long belt coverage in wireless networks. IEEE Transactions on Computers, 64(2):587-592, Feb 2015.

[16] Ling Wang, Lu An, Hao-Qi Ni, Wei Ye, Panos M Pardalos, and Min-Rui Fei. Pareto-based multi-objective node placement of industrial wireless sensor networks using binary differential evolution harmony search. Advances in Manufacturing, 4(1):66-78, 2016. 